Nervenarzt 2011 · 82:695-696

DOI 10.1007/s00115-010-3038-0

(c) Springer-Verlag 2011

\author{
B. Schoser ${ }^{1} \cdot$ S. Zierz ${ }^{2}$ \\ ${ }^{1}$ Friedrich-Baur Institut, Neurologische Klinik Interdisziplinäres Zentrum für \\ Neuromuskuläre Erkrankungen, Klinikum der Universität München, München \\ ${ }^{2}$ Neurologische Klinik Halle, Halle
}

\title{
Neuromuskuläre Erkrankungen
}

Warum ein Themenheft zu neuromuskulären Erkrankungen in Der Nervenarzt?

Das periphere Nervensystem und die neuromuskulären Erkrankungen sind „orphan diseases“ der Neurologie geworden. Trotz vielfältiger klinischer, wissenschaftlicher und v. a. therapeutischer Fortschritte finden auf den großen neurologischen Kongressen Erkrankungen peripherer Nerven und der Muskulatur kaum Beachtung. Ebenso ist auch der Bereich der Motoneuronerkrankungen an vielen Universitäten unterrepräsentiert. Dieses Themenheft soll Ihnen den aktuellen Stand zu Diagnose und Therapie einiger wichtiger neuromuskulärer Erkrankungen zusammenfassen und Ihr Interesse wecken, sich wieder mit diesen immer erfolgreicher behandelbaren Erkrankungen auseinanderzusetzen.

Die Identifizierung der genetischen Grundlage einer zunehmenden Zahl neuromuskulärer Erkrankungen hat das Verständnis und die Klassifikation dieser Gruppe von Erkrankungen in den letzten Jahren deutlich erweitert und verändert. Die Auswahl der vorliegenden Beiträge soll jedoch keine Kurzdarstellung der einzelnen neuromuskulären Erkrankungen sein, sondern soll sich vielmehr auf solche Erkrankungen richten, bei denen die Fortschritte im Verständnis der molekularen Grundlage auch zu einem erweiterten Verständnis der klinischen Phänotypen geführt haben.

Petri u. Meyer beschreiben in ihrer Darstellung die sich diversifizierende Gruppe der neurodegenerativen Motoneuronerkrankungen. Schwerpunkt sind neu gewonnene pathogenetische Erkenntnisse aus molekularbiologischen Untersu- chungen der hereditären Formen. Intraneuronale Proteininklusionen und Aggregatakkumulation stellen das zurzeit ordnende neuropathologische Korrelat dar. Pathogenetisch ursächlich sind hierfür wiederum u. a. Veränderungen in transkriptionsassoziierten DNA/RNA-Bindungsproteinen, die zu Störungen der RNA-Regulation beitragen. Die Aufklärung übereinstimmender Abschnitte der neuronalen Schädigungskaskade ist ein wesentlicher Ansatz zur Entwicklung einer molekulargenetisch-definierten Therapiestrategie sowohl bei der amyotrophen Lateralsklerose (ALS) als auch bei hereditären und sporadischen Vorderhornerkrankungen.

\section{( Die Identifizierung der genetischen Grundlagen veränderte das Krankheitsverständnis}

Obgleich die Myasthenia gravis und das Lambert-Eaton-Rooke-Syndrom die häufigsten Krankheitsbilder aus der Gruppe von Erkrankungen aufgrund einer defekten neuromuskulären Übertragung darstellen, gibt es eine Vielzahl genetisch determinierter Störungen der neuromuskulären Transmission, die teilweise ähnliche Phänotypen aufweisen, dabei jedoch im Unterschied zu den immunogen vermittelten Erkrankungen auf genetisch determinierten Veränderungen der Rezeptorkanäle der postsynaptischen Membranen beruhen. Zusätzlich wird auf neue Aspekte zur doppelseronegativen Myasthenia gravis eingegangen. Dies wird in dem Beitrag von Abicht et al. dargestellt.
Eine der häufigsten Muskelerkrankungen im Erwachsenenalter ist die fazioskapulohumerale Muskeldystrophie (FSHD). Interessanterweise beruht diese Erkrankung nicht auf der Mutation eines spezifischen Genprodukts, sondern auf einer Veränderung regulatorischer nichtkodierender DNA-Abschnitte. In dem Beitrag von Jordan u. Müller-Reible werden die komplexen molekulargenetischen Grundlagen erörtert. Die Möglichkeit einer genetischen Diagnostik bei der FSHD führte weiterhin $\mathrm{zu}$ einem deutlich erweiterten Verständnis des klinischen Phänotyps dieser Erkrankung.

Obgleich den neuromuskulären Erkrankungen in der Vergangenheit der Nimbus einer Unbehandelbarkeit anhing, gibt es nicht nur bei den autoimmunvermittelten Myopathien, sondern selbst bei den progressiven Muskeldystrophien sinnvolle medikamentöse und z. T. auch molekulare Therapiemöglichkeiten. Dies wird in praxisrelevanter Weise in dem Beitrag von Schmidt u. Vorgerd übersichtlich zusammengefasst. Wir hoffen, Ihnen mit der Auswahl dieser Themen einen Eindruck von der interessanten Komplexität und Vielfalt neuromuskulärer Erkrankungen vermitteln zu können.

Wie geht es weiter?

Als zielführend werden die Translation der molekularen Pathogenese in moderne Therapieansätze wie z. B. für die Enzymersatztherapie bei metabolischen Erkrankungen oder die „Exon-skipping“-Studie bei Duchenne-Muskeldystrophie anzusehen sein $[1,2,3]$. Vielfältige Strategien zur „trial readiness“ liegen u. a. in der Etablierung neuer nationaler und internatio- 
naler Netzwerkstrukturen, in Patientenregistern, der Etablierung von Studienzentren sowie der Einbeziehung spezialisierter Selbsthilfegruppen. Dieses strategisch sinnvolle Konzept konnte schon für die Muskeldystrophien (www.md-net.de) und die mitochondrialen Erkrankungen (www.mitonet.de) erfolgreich in den letzten Jahren umgesetzt werden.

\section{(7) Die Translation der Molekularpathogenese in Therapieansätze ist ein wesentliches Ziel}

Die fortlaufende Erforschung der Molekularpathogenese wird vielfältige therapeutische Wege für Patienten mit neuromuskulären Erkrankungen eröffnen. Es lohnt sich also für junge wissenschaftlich engagierte Neurologen, Zeit, Lust und Freude in die Auseinandersetzung mit neuromuskulären Erkrankungen zu investieren.
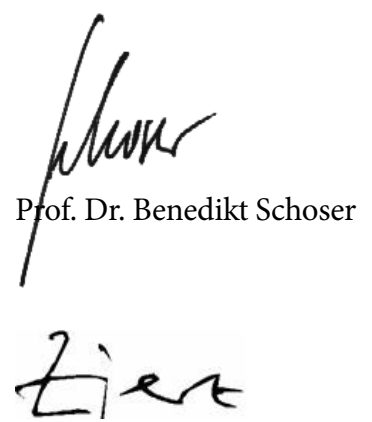

Prof. Dr. Stephan Zierz

\section{Korrespondenzadresse}

\section{Prof. Dr. B. Schoser}

Friedrich-Baur Institut, Neurologische Klinik Interdisziplinäres Zentrum für Neuromuskuläre Erkrankungen, Klinikum der Universität München Ziemssenstr. 1a, 80336 München bschoser@med.uni-muenchen.de

\section{Literatur}

1. Aartsma-Rus A, den Dunnen JT, van Ommen GJ (2010) New insights in gene-derived therapy: the example of Duchenne muscular dystrophy. Ann N Y Acad Sci 1214:199-212

2. Goemans NM, Tulinius M, van den Akker JT et al (2011) Systemic administration of PRO051 in Duchenne's muscular dystrophy. N Engl J Med 364:1513-1522

3. van der Ploeg AT, Clemens PR, Corzo D et al (2010) A randomized study of alglucosidase alfa in lateonset Pompe's disease. N Engl J Med 362:13961406

\section{Erste europäische Tourette- Therapiestandards}

Kürzlich veröffentlichte Leitlinien setzen erstmalig europaweite Standards für die Therapie von Kindern und Jugendlichen mit Tic-Störungen. Die Springer-Fachzeitschrift „European Child \& Adolescent Psychiatry" gibt ein Schwerpunktheft mit Online-Zugriff heraus.

Bei etwa zehn Prozent aller Kinder und Jugendlichen treten nicht kontrollierbare Bewegungen und Lautäußerungen, sogenannte Tics, auf. Zumeist sind diese folgenlos und sie verschwinden innerhalb eines gewissen Zeitraumes. In wiederum zehn Prozent dieser Fälle wird der Tic allerdings chronisch, eine schwere Form dieser Störung ist das sogenannte Tourette-Syndrom. Zur Behandlung der Betroffenen gibt es nun Leitlinien, die Ärzten, Psychiatern bzw. Psychotherapeuten die Möglichkeit geben, die Störung eindeutiger zu erkennen und gleichzeitig mehr über die geeigneten Therapien zu erfahren. Die Leitlinien sind von der „European Society for the Study of Tourette Syndrome" (ESSTS) verabschiedet worden und sind veröffentlicht in „European Child \& Adolescent Psychiatry", einer Fachzeitschrift von Springer Medizin.

„Mit den Leitlinien bekommen Fachleute in Europa nun umfassendes Wissen an die Hand," erklärt Professor Veit Rößner, Tourette-Spezialist an der Universität Dresden. „Benötigen manche lediglich eine fachgerechte Anleitung, wie sie mit ihrer Störung umgehen lernen," führt er weiter aus, ,, so muss in mittelschweren bis schweren Fällen, bei denen die Lebensqualität oder die schulischen Leistungen stark beeinträchtigt sind, mit medikamentösen bzw. selten auch mit neurochirurgischen Therapien gearbeitet werden. Wir haben außerdem erste Studien auf den Weg gebracht, deren Ergebnisse den Einsatz von Psychopharmaka mit moderneren Wirkstoffen für schwer Betroffene befürworten." Die Leitlinien sind unter Federführung von Veit Rößner entstanden, der außerdem Mitglied im Herausgeberboard der Zeitschrift „European Child \& Adolescent Psychiatry" ist.
Das Schwerpunktheft der „European Child \& Adolescent Psychiatry“ ist über das Online-Archiv SpringerLink verfügbar, Teile davon sind als Open Access Artikel frei zugänglich. Die Publikation von Springer Medizin ist Europas einzige Zeitschrift mit Peer-Review-Verfahren im Bereich der Kinder- und Jugendpsychiatrie und ist bei Medline, ISI sowie allen wichtigen Datenbanken gelistet. Ziel der Zeitschrift ist es, mit ihren Beiträgen das Verständnis der Psychopathologie von Kindern und Jugendlichen zu fördern. Dabei legt sie großen Wert auf empirische Forschung mit klinischer Relevanz. 